\title{
A DFT Calculation: Toxicity of Water Soluble Arsenicals
}

\author{
M. A. Salam \\ Industrial Physics Section, Bangladesh Council of Scientific and Industrial Research (BCSIR), \\ Chittagong Cantonment-4220, Bangladesh
}

\begin{abstract}
We have used the density functional theory to make a predictive toxicity model of water soluble arsenicals. The structures are optimized for the minimum energy of the Schrödinger equation. From this optimized structure, we obtain the bond distance and angles of the stable structures. We have calculated vibrational frequencies, Ionization potential, atomic charges, electrophilicity and electron affinity of those arsenicals. The calculated vibrational frequencies and other physical properties are compared with those found from the different experiment. The mode of vibrational frequencies, electrophilicity and atomic charges (Mulliken) calculated for MMA(III), DMA(III) ,arsenic acid, arsenous acid, MMA(V) and DMA(V) that follows acute toxicity of those arsenicals in laboratory animals. Raman micro-spectroscopy might also be showed its potential applications in the toxicology screening of chemicals and new biomaterials, with a range of cell types. Applying the model one could predict toxicity level of unknown water soluble arsenicals in future.
\end{abstract}

Keywords : DFT, Raman spectroscopy, toxicity, Water soluble arsenicals $\mathrm{LD}_{50}$

\section{Introduction}

Toxicity of a substance is a measure of its harmful effects on living organisms (Lu,1996). Basically, toxicity may be categorized into three types, viz., chemical, biological and physical toxicity $(\mathrm{Lu}, 1996)$. Both inorganic and organic chemicals may show toxic effects on living beings, for example, inorganic substance such as lead, chlorine gas and hydrofluoric acid and organic compounds such as methyl alcohol cause damaging effects on living organisms. Bacteria and viruses are the primary entities that induce diseases into plants and animals, including human beings. Various harmful incidents to the living systems such as concussion, inhalation of toxic chemicals and ionization radiation, e.g., $\mathrm{X}$-rays or various radioactive rays $(\alpha, \beta$ and $\gamma)$ may be considered as physical toxicity. Toxicity of metals and their various derivatives play dominant role in damaging or having a deleterious effect on organisms (Seiler et al, 1988). The importance of some heavy toxic metals e.g., arsenic, lead, mercury and cadmium is remarkable. Among these, arsenic toxicity has attracted a large amount of attention from researchers due to its grievous effect and at the same times its usefulness towards living beings.

Arsenic is a metalloid found in water, soil and air from natural and anthropogenic sources. It exists in inorganic and organic forms and in different oxidation states $(-3,0+3,+5)$.

\footnotetext{
* Corresponding author: E-mail: salam.bcsir@gmail.com
}

In the case of environment exposure, toxicologist is primarily concerned with arsenic in the trivalent and pentavalent oxidation state. Fig-1 shows the structure of several water soluble arsenicals of toxicological relevance. The more commonly known arsenic compounds, arsenate and arsenite are the anionic forms of arsenic acid and arsenous acid, respectively. Monomethylarsenic acid $\left(\mathrm{MMA}^{\mathrm{v}}\right)$ and dimethylarsenic acid $\left(\mathrm{DMA}^{\mathrm{v}}\right)$ are stable methylated mammalian metabolites of inorganic arsenic and are primarily exerted in the urine (ToxFAQs ${ }^{\mathrm{TM}}, 2007$ ).

An interesting event is that DMAv and sodium salt of MMA have been used as herbicides. Arsenic is generally more toxic in its inorganic state compared with its organic form (Petrick et al, 2001), e.g., methylation of trivalent inorganic arsenic to form a pentavalent organic arsenic decreases the degree of toxicity (Chris et al, 2006). Although arsenic has some usefulness in human safety and civilization, e.g., as a pesticide, preserving or pressure-treating wood, producing glass, in copper and other metal manufacturing, in the electronics industry as well as in the medical science, it is more familiar due to its poisonous effects on living beings, e.g., thickening and discoloration of skin, damaging blood vessels, abnormal heart rhythm and blindness including carcinogenicity (Seiler et al, 1988, Petrick et al, 2001).Therefore, 


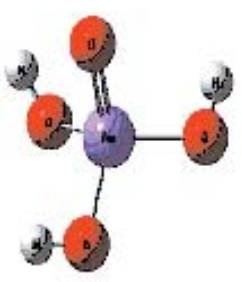

Arsenic acid

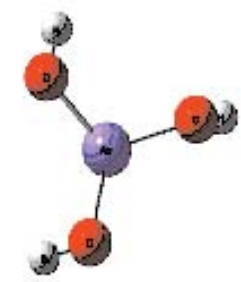

Arsenous acid

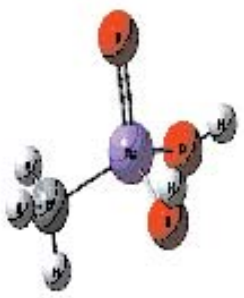

MMA (V)

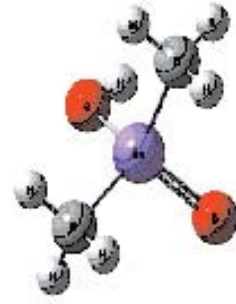

DMA (V)

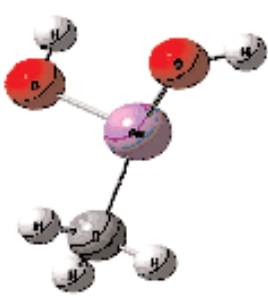

MMA(III)

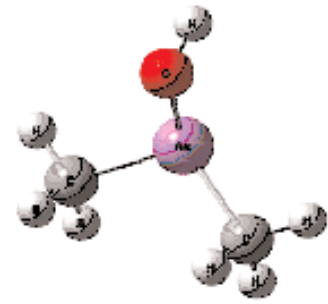

DMA(III)

Fig. 1: The structure of water soluble toxic arsenicals

knowledge of its degree of toxicity along with that of its various derivatives has become essential to maintain environmental safety and security. Experimental determination of toxicity of these water soluble arsenicals is difficult due to time, cost and availability of sources associated with it. In this regard, theoretical modeling has been developed to explain the toxicity of these arsenicals in terms of their vibrational frequency and structural parameters, e.g., geometric, topological, electronic and quantum chemical etc.

Raman spectral analysis is capable of supplying more detailed information about the entire biochemical composition of a cell than traditional assays (such as MTT or LDH) in a shorter period of time (Krafft 2004, Notingher et al, 2004). Raman spectroscopy, when combined with the analytical methods presented, is a powerful tool for cellular analysis and in vitro toxicological testing on human cells. It offers the opportunity to enhance the understanding of how chemicals react with human cells in real time and may find applications in the toxicology screening of other drugs, chemicals and new biomaterials (Parr et al, 1989), with a range of cell types. This research also supports the goal of an alternative testing system that reduces economic cost and increases throughput without animal testing, as stipulated by the EU [(E.C.), 2001].

\section{Computational details}

The total energies and the atomic/molecular orbital energies ( $\psi_{\text {HOMO }}$ and $\left.\psi_{\text {LUMO }}\right)$ of all water soluble toxic arsenicals have been calculated at the B3LYP level of theory (Chattaraj PK 2009, Chattaraj et al, 2009 ,Gaussian 09W) with 6$31+\mathrm{G}[\mathrm{d}]$ basis set using Gaussian 09W program package (Mulliken,1995). Calculation has been performed for the water soluble arsenicals like arseneous acid, arsenic acid, MMA (III), DMA (III), MMA (V) and DMA (V) as well. In all cases, optimum geometry has been obtained along with the vibrational frequency at the B3LYP/6-31+G[d] level of theory using Gaussian 09W. All the necessary atomic charges have been calculated using the Mulliken population analysis (MPA) (Parr, 1999). Electrophilicities (Chattaraj et al 2009, Petrick et al 2000) have been calculated with help of electron affinity and ionization potential of the concerned optimized structure.

\section{Water soluble arsenicals}

(i). Arsenic acid: it is possible to optimize arsenic acid in the shape of arsenic tetrahedral. The As-O bond distance is found to be $1.8700 \AA$. The vibrational frequency (intensity) is calculated to be $3924.94 \mathrm{~cm}^{-1}(6.7 \mathrm{~km} / \mathrm{mol})$ and scattering activity $172.168\left(\mathrm{~A}^{4} / \mathrm{AMU}\right)$. Electron affinity and Dipole

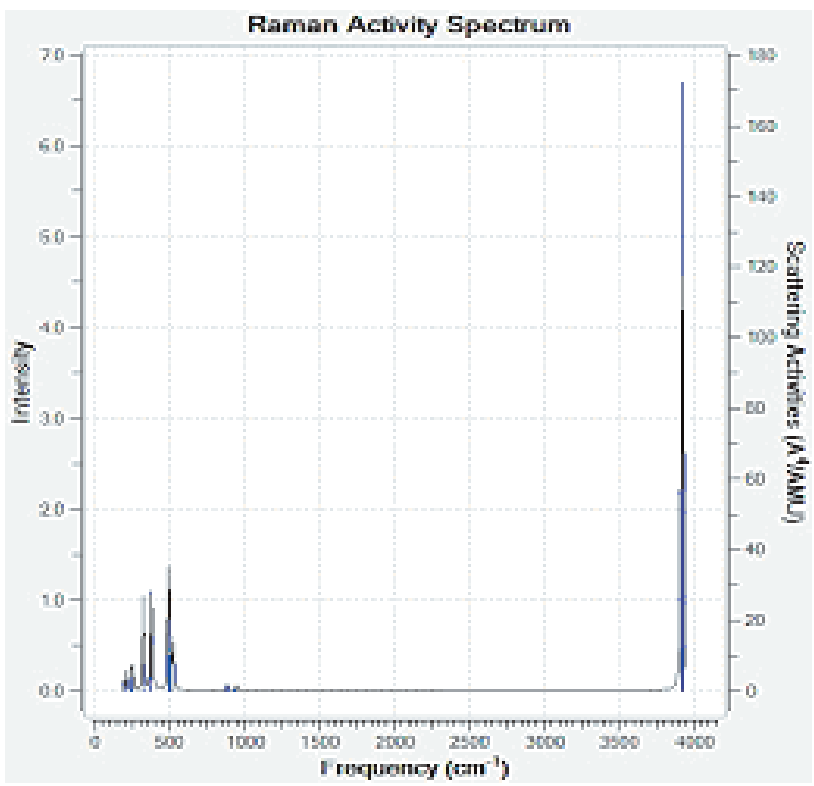

Fig. 2: The Vibrational spectrum of Arsenic acid 
moment are -0.1147 and 2.0799 respectively. The Mulliken atomic charge is -1.202 . Although IR frequency (intensity) is $499.469 \mathrm{~cm}^{-1}$ (1200 epsilon).The computed vibrational spectrum has been shown in fig-2

(ii) Arsenous acid: In this optimized configuration the bond distance of As-O is found to be $1.8700 \AA$. Electron affinity and dipole moment are 0.03749 and 2.78 respectively. Mulliken atomic charge of the molecule is -0.857 . Rational Infrared frequency is $524.362 \mathrm{~cm}^{-1}$ which epsilon value 700 . It has strong vibration whose frequency and intensity is $3910.22 \mathrm{~cm}^{-1}$ and $7.99 \mathrm{~km} /$ mole. The vibrational spectrum calculated from the first principles has been shown in fig-3.

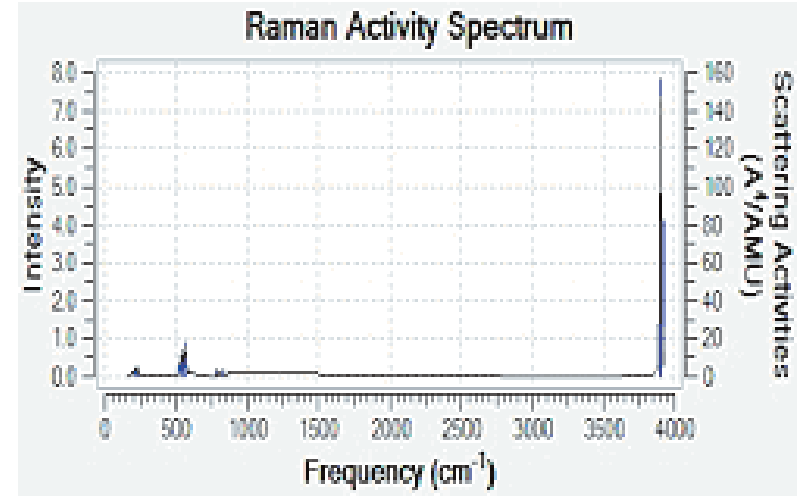

Fig. 3: The Vibrational spectrum of arsenous acid

\section{Monomethylarsenous acid [MMA (III)]}

In this cluster the bond distance of As-O and As-C are 1.8700 $\AA$ and $1.9800 \AA$ respectively. Electron affinity and dipole moment are -0.01265 and 1.5099. Mulliken Atomic charge of the calculated geometry is 0.718 . The highest frequency of IR is $541.67 \mathrm{~cm}^{-1}$ where epsilon value is 450 . The calculated spectrum of MMA (III) has been shown in fig-4.The complex has several vibrational modes of frequencies and those are 3907.22(4.65), 3393(2.85), 3275.65(4.8), $1470.56(0.5), 716.31(.1), 502(0.88) \mathrm{cm}^{-1}(\mathrm{~km} / \mathrm{mol})$. This is an example of strong internal vibrations within the system.

\section{Dimethylarsenous acid [DMA (III)]}

The trigonal planer geometric shape of DMA (III) has been optimized. The bond length of As-O is $1.8700 \AA$ and As-C is $1.9800 \AA .0 .00851$ and 1.8355 are the electron affinity and dipole moment. Mulliken atomic charge is 0.717 . The highest frequency of IR is $562.40 \mathrm{~cm}^{-1}$ where epsilon value is 265. In fig-5 the spectrum of DMA (III) shows several fre- quency modes near most like MMA (III). Remarkable frequency of this cluster are 3899.6(5.25), 3391.16(4.1), $3269.1(7.1), 1445.32(0.85)$ and $500.64(2.18) \mathrm{cm}^{-1}(\mathrm{~km} / \mathrm{mol})$.

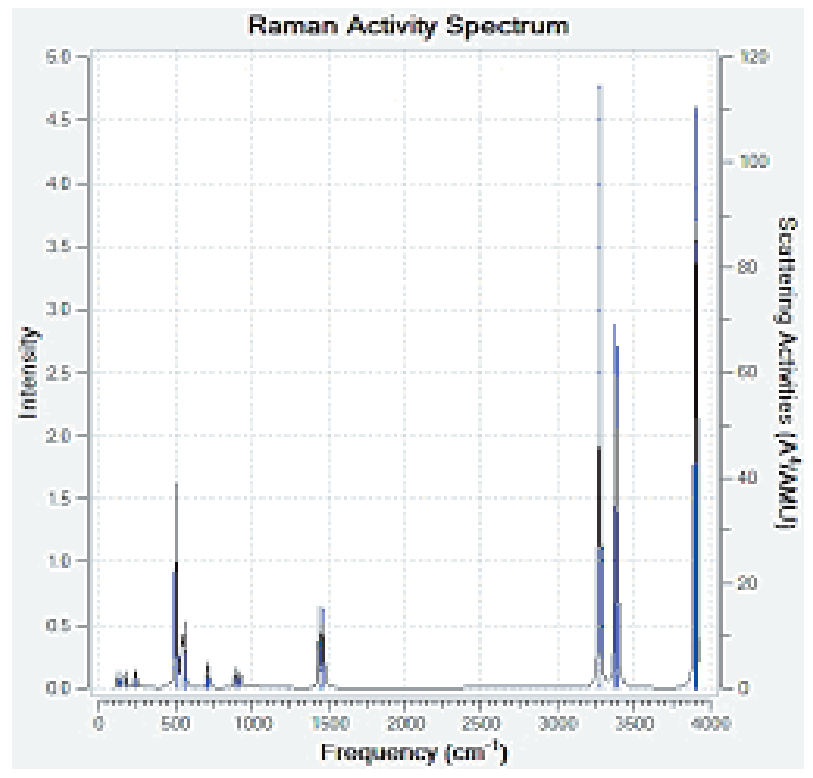

Fig. 4: The vibrational spectrum of MMA(III)

\section{Monomethyl arsenic acid [MMA (V)]}

In this optimized geometry one double bond with $1.8700 \AA$ bond length between As-O and As-C bond distance is $1.9800 \AA$. The Dipole moment and electron affinity are 3.0573 and 0.06202 accordingly. Muliken atomic charge is -

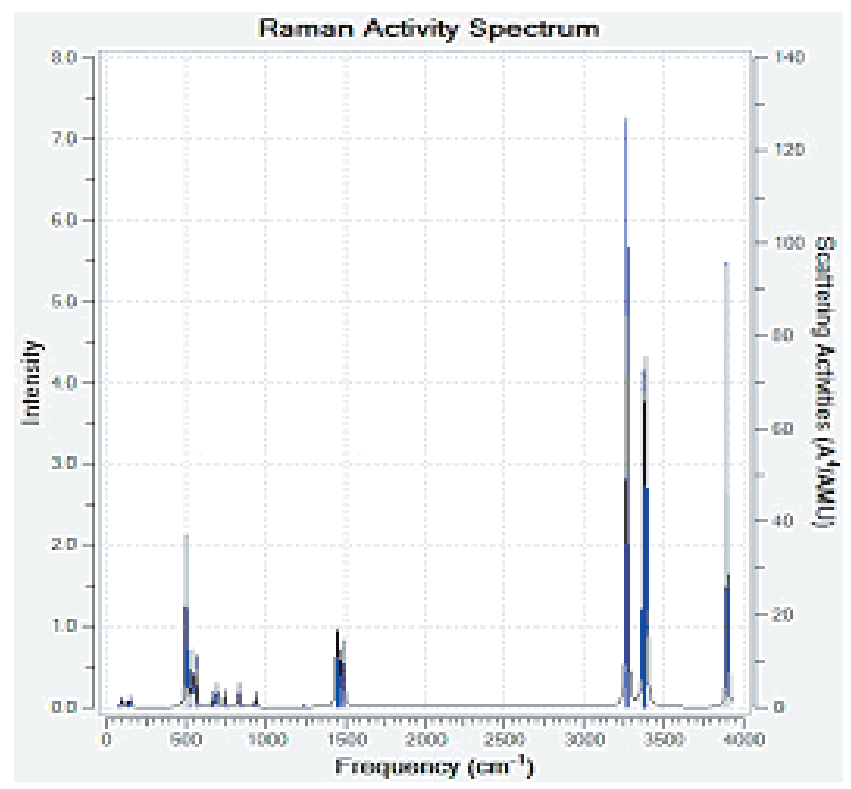

Fig. 5: The vibrational spectrum of DMA(III) 
0.886. Infra red frequency of this geometry shows 521.168 and epsilon value is 700 . Significant vibrational frequencies (Intensities) are 3917(7.95), 3293.72(5.2), 3386.45(3.1), 1465.66(0.6), 484.81(1.1), 404.233(0.9) and 314.306(.5).

\section{Dimethyl arsenic acid[DMA (V)]}

The bond distance of As-O and As-C are 1.8700 $\AA$ and $1.9800 \AA$ respectively and have double bond with bond distance $1.9800 \AA$. Electron affinity and dipole moment are 0.02196 and 4.7472. Mulliken Atomic charge of the calculated geometry is 0.881 . IR highest frequency is $533.461 \mathrm{~cm}^{-1}$ where epsilon value is 330 . The calculated spectrum of DMA (V) is shown in fig-7.The complex has several vibrational modes the frequencies of which are 3282.58(7.3), 3399.98(5.0), 3911.05(5.05), 1464.64(1.3), 487.907(2.2), $117(0.90) \mathrm{cm}^{-1}(\mathrm{~km} / \mathrm{mol})$.

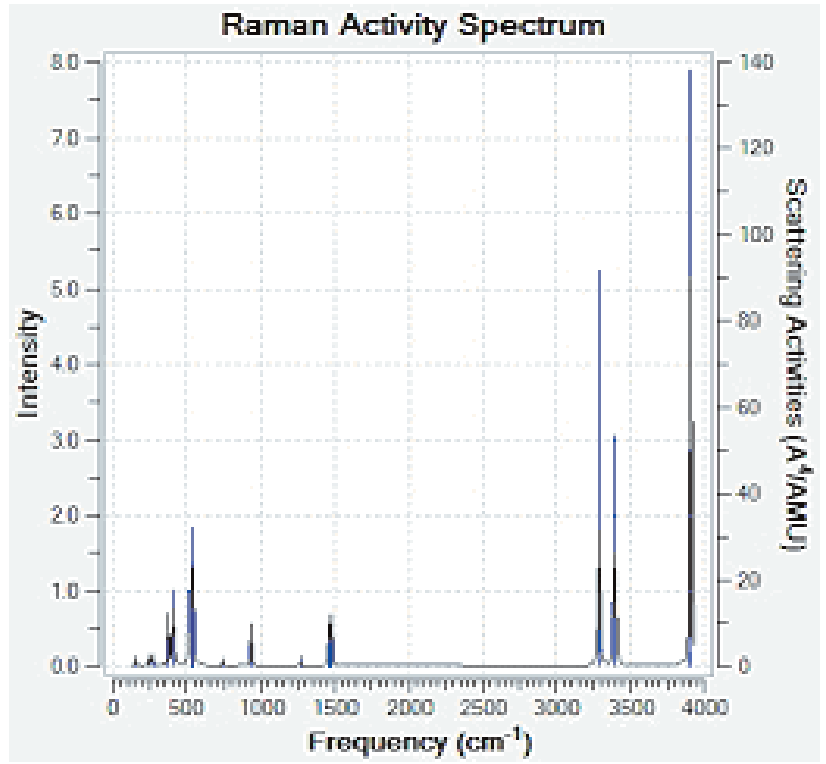

Fig. 6: The Vibrational spectrum of MMA(V)

\section{Results and Dicussion}

The degree of harmful effect due to highly toxic arsenicals is important information required for environmental safety. In this regard, the potential of the mode of vibrational frequencies (Raman spectroscopy), electrophilicity ( $\omega)$ and atomic charge (Q) have been investigated in the following subsections.

\section{Acute toxicity}

The acute toxicity of arsenic is related to its chemical form and oxidation state. The $\mathrm{LD}_{50}$ values of several arsenicals in laboratory animals have been displayed in Table I (Styblo et $a l, 2000)$. A basic tenet is that the acute toxicity of trivalent arsenic is greater than pentavalent arsenic (Styblo et $a l, 1997]$. For example, in the mouse, the oral $\mathrm{LD}_{50}$ of arsenic trioxide is more than 36-fold lower than that of MMA (V). In

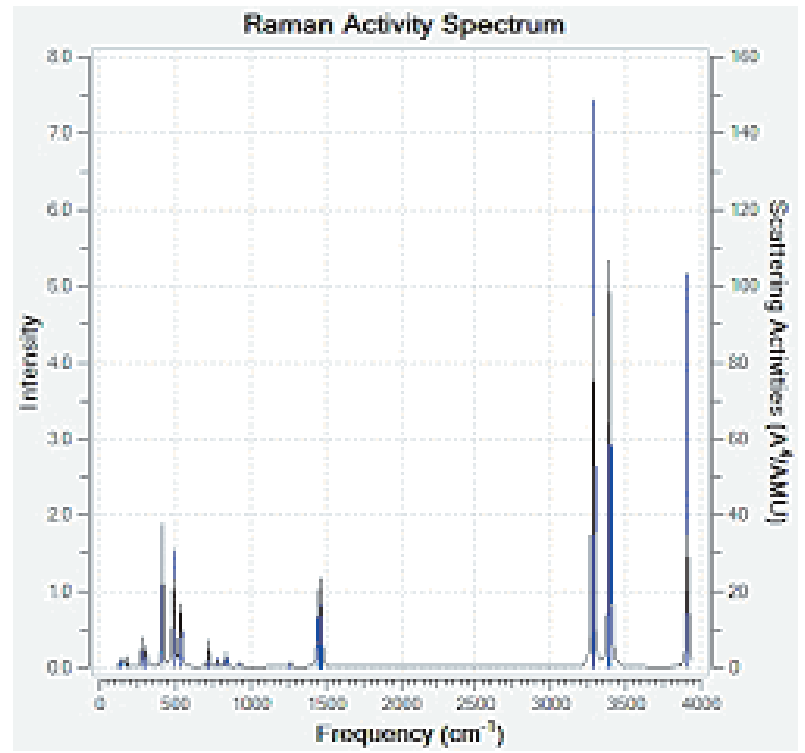

Fig. 7: The vibrational spectrum of DMA(V)

the human adult, the lethal range of inorganic arsenic is estimated at a dose of 1-3 mg As/ $\mathrm{kg}$ (Ellenhorn, 1997). The characteristics of severe acute arsenic toxicity in human include gastrointestinal discomfort, vomiting, diarrhoea, bloody urine, anuria, shock, convulsions, coma and death.

For many years it was believed that the acute toxicity of inorganic arsenic was, greater than organic arsenic and hence, the methylation of inorganic arsenic was a detoxification reaction. This dogma was held because DMA (V), the primary excreted metabolite of inorganic arsenic. However, Cullen et al. (1989) found that a derivative of MMA (III) was more than arsenite to the microorganism Candida humicola in virto (Styblo et al,1999). Human cells are also more sensitive to the cytotoxic effects of MMM (III) than arsenite (Styblo, 1995, Verstovsek, 2004,Petrick et al., 2000; Styblo et al., 1999, 2000). DMA (III) behaves at least as arsenite in several human cell types (Styblo et al., 2000). Recently Petrick et al. (2001) reported that MMA (III) has a lower $\mathrm{LD}_{50}$ than arsenite in the hamster (Michael, 2002). The 
greater acute toxicity of the methylated trivalent intermediates of arsenic suggests that the methylation of arsenic is not solely a detoxification mechanism.

\section{Toxicity level prediction using computed parameter}

A set of total six water soluble toxic arsenicals were selected [Table I] as experimental objects to estimate the degree of toxicity. Table II presents the atomic energy, electrophilicity and mulliken charges values with the experimental and calculated toxicity values in terms of $\mathrm{LD}_{50}$. arsenic are more toxic compared with the organic one in general, both types have harmful effects on organisms, and therefore estimation of their degree of toxicity is important. The corresponding regression model has been shown in fig- 8

A hypothesis can be made based on analytical observation on mode of vibration, Raman activity and intensity of the frequency from figures [fig-2, 3, 4, 5, 6, 7] of calculated arsenicals. According to this hypothesis, Raman activity spectrum may be expressed the toxicity level for the water soluble toxic arsenicals. For MMA (III), it has been observed that

Table I: Acute toxicity of arsenic in laboratory

\begin{tabular}{l|c|c|c|c}
\hline Chemical & Species & Rout & LD $_{50}(\mathrm{mg} / \mathrm{kg})$ & Reference \\
\hline MMA $^{\mathrm{III}}$ & Hamster & ip & 2 & Petrick et al. $(2001)$ \\
DMA $^{\mathrm{III}}$ & Hamster & ip & 6 & Petrick et al. $(2001)$ \\
Arsenite & Mouse & im & 8 & Petrick et al. $(2001)$ \\
Arsenate & Mouse & im & 22 & Bencko et al. $(1978)$ \\
MMA $^{\mathrm{V}}$ & Mouse & Oral & 916 & Kaise et al. $(1989)$ \\
DMA $^{\mathrm{V}}$ & Mouse & Oral & 648 & Kaise et al. $(1989)$ \\
\hline
\end{tabular}

Two parameter regression models have been applied using the electrophilicity index and atomic charges. The regression model for this experiment is as follows:

Calc. $\mathrm{LD}_{50}=152.22 \times 10^{3} \times(10.17)^{\omega / .2} \times(\mathrm{Q} / .2)^{9.49}$

Although electrophilicity and mulliken atomic charge individually have a good predictive potential that show a singular significance. Arsenic Compound having less electrophilicity index value usually shows higher level of toxicity. On the contrary highly mulliken atomic charged arsenic compound shows lower level of toxicity. Therefore, electrophilicity and atomic charge have been applied to measure the toxicity of those arsenicals. It has been found that MMA (III) shows more toxicity $\left(\mathrm{LD}_{50}=2.099\right)$ and MMA (V) less toxicity $\left(\mathrm{LD}_{50}=940.662\right)$, as expected from literature (In order, not in quantity).

The derivatives of the arsenic exist in both the inorganic and organic forms in the environment. Although inorganic

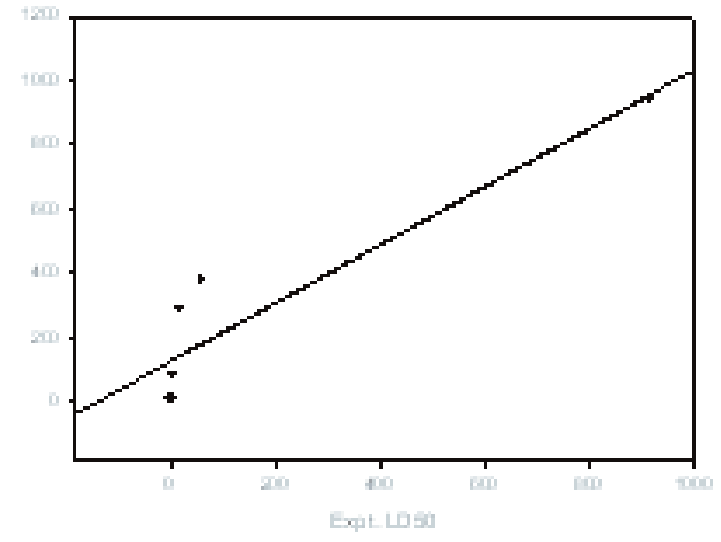

Fig. 8: Experimental verses calculated toxicity $\left(L_{50}\right)$ values using two parameter regression models of arsenicals

the value of intensity (4.8) and Raman activity (114.53) for the significant frequency is low where as for MMA (V), the value of intensity (7.95) and Raman activity (139) is high. It

Table II: Atomic energy(E), electrophilicity index $(\omega)$ and experimental and calculated $\mathrm{LD}_{50}$ values for water soluble toxic arsenicals

\begin{tabular}{l|c|c|c|c|c}
\hline Chemical & $\mathrm{E}(\mathrm{au})$ & $\omega(\mathrm{eV})$ & $\mathrm{Q}$ & Expt. $\mathrm{LD}_{50(\mathrm{mg} / \mathrm{kg})}$ & Calculated $\mathrm{LD}_{50}$ \\
\hline MMA $^{\mathrm{III}}$ & -2425.29 & 0.0619 & -0.718 & 2 & 2.099 \\
DMA $^{\mathrm{III}}$ & -2389.38 & 0.0646 & -0.721 & 6 & 3.127 \\
Arsenous acid & -2461.21 & 0.0936 & -0.857 & 8 & 77.125 \\
Arsenic acid & -2536.45 & 0.1073 & -0.860 & 22 & 280.173 \\
MMA $^{\mathrm{V}}$ & -2500.49 & 0.1271 & -1.031 & 916 & 940.662 \\
DMA $^{\mathrm{V}}$ & -2464.58 & 0.1114 & -0.890 & 648 & 373.100 \\
\hline
\end{tabular}


is remarkable that the lowest toxic compound shows highest intensity on the contrary highest toxic compound shows lowest intensity. Again mode of vibration and Raman activity may also lead a good contribution to assume the level of toxicity though they have no singular behaviour on that.

\section{Conclusion}

Atomic charge (Q) and electrophilicity index $(\omega)$ are capable of explaining the level of toxicity of water soluble arsenicals. The toxicity of different arsenic compound and also toxicity of different oxidation states of an element can be predicted using this model. Raman activity spectrum can be used to explain and predict the level of toxicity of various arsenic compounds. The result of this research would be helpful to get important information for environmental safety and to make a toxicity meter of water soluble arsenicals.

\section{References}

Chattaraj P. K. (2009). Chemical reactivity theory: A density functional view. Taylor \& Francise/CRC Press, Boce Raton, FL XVIII+576pp/SBN:987-1-4200-6542.

Chattaraj P. K. and Giri S. (2009). Electrophilicity index within a conceptual DFT framework. Annu rep prog. Chem Sect C, DOI 10:1039/b802832j.

Chris A. Owen Jamuna Selvakumaran, Ioan Notingher, Gavin Jell, Larry L. Hench, and Molly M. Stevens (2006). In Vitro toxicology evaluation of pharmaceuticals using Raman micro-spectroscopy, Journal of Cellular Biochemistry 99: 178-186.

Gaussian 09W (2009). Rev:A. 02 Front:6.1,multiprocessor version; Gaussian, Inc., Wallingford, CT 06492 USA.

Krafft C. (2004). Bioanalytical applications of Raman spectroscopy, Anal Bio analytical Chem 378: 60-62.

Lu F. C. (1996). Basic toxicology: fundamentals, target organs, and risk assessment, 3rd edn. Taylor and Francis, Washington.

Mulliken R. S. (1995). Electronic population analysis on LCAO-MO molecular wave functions I. J Chem Phys 23: 1833-1840.dio:10.1063/1.1740588.

Michael F. Hughes (2002). Arsenic toxicity and potential mechanism of action, toxicological letters 133: 1-16

Notingher I, Green C, Dyer C, Perkins E, Hopkins N, Lindsay C and Hench L. L. (2004b). Discrimination between ricin and sulphur mustard toxicity in vitro using Raman spectroscopy, $J$ R Soc Interface 1:79-90.
Petrick J. S., Jagadish B., Mash E. A. and Aposhian H. V. (2001). Monomethylarsenous acid [MMA(III)] and arsenite : $\mathrm{LD}_{50}$ in hamsters and in vitro inhibition of pyruvate dehydrogenase. Chem Res Toxicol 14:-651656. Doi: 10.1021/txooo264z.

Parr R. G. and Yang W. (1989). Density functional theory of atoms and molecules. Oxford University Press, New York

Parr R. G., Laszlo V., .Szentpaly and Shubin Liu (1999). Electrophilicity Index, J. Am. Chem. Soc. 121: pp1922-1924.

Petrick J. S., Ayala-Fierro F., Cullen,W. R., Carter, D. E. and Aposhian H. V. (2000). Monomethylarsnous acid MMA(III) is more than arsenite in chang human hepatocytes. Toxicol.Appl.Pharmacol. 163: -203-207.

Styblo M., Del Razo L. M., Vega L.,Germolec D. R., LeCluyse E. L., Hamilton G. A., Reed W., Wang C., Cullen W. R. and Thomas D. J. (2000). Comparative toxicity of trivalent and pentavalent inorganic and methylated arsenicals in rat and human cells. Arch.Toxicol. 74: 289-299.

Styblo M., Serves S. V., Cullen W. R. and Thomas D. J., (1997). comparative inhibition of yeast glutathione reductase by arsenicals and arsenothiols.Chem.Res. Toxicol. 10: -27-33.

Styblo M., Vega L., Germolec D. R., Luster M. I., Del Raza L. M., Wang C., Cullen W. R. and Thomas D. J.,(1999). Metabolism and toxicity of arsenicals in cultured cells. In:Chappell, W.R., Abernathy, C.O.,Calderon, R.L(Edn.), Arsenic exposure and health effects.Elsevier, New,York, pp 311-323.

Styblo M., Yamauchi H. and Thomas D. J., (1995). Comparative in vitro methylation of trivalent and pentavalent arsenicals., Toxicol. Appl. Pharmacol. 135: 444-449.

Seiler H. G, Sigel H. and Sigel A. (1988). Handbook on toxicity of inorganic compounds, Marcel Dekker, New York.

ToxFAQs TM for Arsenic (2007). Agency for toxic substances and disease registry (ATSDR) Atlanta, GA:US Department of Health and Human Services, public Health Service.

Verstovsek Z. E. (2004). Arsenic derivatives as therapeutic agents for hematologic malignancies, Leuk res 28,pp 901-903.dio:10.1016/j.leukers.2004.01.013.

Received:June 03, 2010;

Accepted : November 09, 2010 